\title{
Open Grid Services Architecture
}

\author{
Jem Treadwell \\ Hewlett-Packard
}

\begin{abstract}
A decade after taking its first steps as a research project, grid computing is on the road to "pervasive adoption" across all types of organization, from the campus to the enterprise data center. While many forms of grid infrastructure exist today, some proprietary and some based on open protocols and software components, the key to continued progress in the evolution of grid computing is standardization. By building grids and applications on standards-based components, architects can meet their organizations' needs with confidence that they are interoperable with other standards-based grids, and that they can grow and adapt as those needs change. The Global Grid Forum's (GGF) Open Grid Services Architecture (OGSA(tm)) describes a service-oriented grid architecture that addresses standardization by defining a set of foundational capabilities for interoperable grids. The OGSA working group publishes the architecture framework and related documents. In this session the speaker will: * Discuss the evolution of grid computing; * Explain how a service-oriented grid fits with other leading-edge enterprise technologies; * Provide details of OGSA and its core capabilities, including progress and plans.
\end{abstract}




\section{Q\&A - Jem Treadwell}

\section{Questioner: G. Allen}

How will scientific applications interact with OGSA?

\section{Jem Treadwell}

The question related to an existing application and to the use of the Simple API for Grid Applications (SAGA) being specified by the Open Grid Forum's SAGA Research Group. In some cases, OGSA services such as the Basic Execution Service (BES) will invoke legacy applications without change, other than perhaps the use of simple wrapper scripts. OGSA services will be specified with Web service interfaces, so where direct interaction is needed these interfaces will need to be invoked. While it may not be practical for legacy applications to become Web service clients, it does seem reasonable for SAGA or other intermediate APIs to handle the necessary protocol exchanges.

I would strongly encourage SAGA-RG and any group that is concerned with interfacing with OGSA to contact Hiro Kishimoto, the OGSA Working Group co-chair, to arrange a joint meeting.

\section{Questioner: D. Gannon}

Great job presenting OGSA! When do you see implementations of OGSA emerging? Will H.P. implement a version of OGSA?

\section{Jem Treadwell}

I think it will take another year or so for OGSA to evolve to a point where commercial products are based on it. Although the OGSA WSRF Basic Profile is close to publication, the higher-level service specifications and profiles such as HPC, Data, and Execution Management must be completed before it can be applied in practical applications. The various groups developing these are making good progress, but standards work always takes time.

The specifications and profiles will arrive gradually, and developers will implement them alongside their existing interfaces-so the move to a fully OGSA-compliant infrastructure will be a gradual migration, rather than the sudden appearance of a full OGSA suite.

HP's grid solutions are based on a combination of internally developed components and open-source and partner products. As the various OGSA specifications arrive l'd expect that we'll adopt them in our own products where they make sense and where our customers have a need for them, and we'll encourage our partners to adopt applicable standards as they become available. 


\section{Questioner: A. Trefethen}

Could you say more about the convergence of the conflicting standards? Do you anticipate further basic profiles?

\section{Jem Treadwell}

The WSRF Basic Profile was so named because there was a strong desire within the GGF community that OGSA should not be constrained to a WSRFbased stack, and we were expecting to develop a WS-Management based profile. However, given the recent announcement from Microsoft, HP, IBM \& Intel that they are working on a converged stack, there is currently no activity on a WS-Management profile. Once the converged stack is published I would anticipate that we would revise the OGSA Basic Profile, but bear in mind that the convergence path has been designed so that no refactoring will be required for work based on either WSRF or WS-Management.

\section{Questioner: W. Gropp}

Experiences with languages show that the best are designed by 1 or $11 / 2$ people to give a simple, uniform, consistent vision. OGSA seems to be the opposite. What is or can be done to ensure that the parts of OGSA are consistent?

\section{Jem Treadwell}

Standards work is usually the work of a broad-based committee, although sometimes a standard is based on work donated by a small group or something that is already widely adopted, either of which can make the process smoother and faster, and maybe yield a better result. OGSA is farreaching, but its components - e.g. BES, Data Management, HPC - are being developed by focused working groups, often driven by a core of three or four people. The OGSA group frequently holds joint meetings - both telecons and face-to-face meetings - with those groups, to discuss the integration and ensure that we have consistent goals.

\section{Questioner: A. Trefethen}

I am interested to see that one of the first profiles is HPC. Are the follow-on profiles listed as a wish list or have they been identified by specific groups who will drive then?

\section{Jem Treadwell}

The HPC profile was proposed by Marvin Themer of Microsoft, to address a specific product need. Marvin is driving the work, and it's making rapid progress. The OGSA Security profiles, which address secure communication, have been developed within the OGSA-WG, and are nearing publication. The other proposed profiles will follow completion of the underlying service specifications, and will be developed by the working groups developing those specifications. 